\title{
LA NÉGOCIATION DE LA FORME : STRATÉGIE ANALYTIQUE EN CLASSE D'IMMERSION ${ }^{1}$ \\ Roy Lyster, Université McGill
}

\begin{abstract}
This article proposes that the negotiation of form could play a beneficial role in immersion pedagogy. As an analytic strategy, the negotiation of form differs from the negotiation of meaning in that it requires students to go beyond the stage of simply getting their meaning across. Students are pushed to produce more accurate and more appropriate utterances in a way which allows them to draw actively on their own (socio)linguistic resources. Examples of such a strategy are provided from a Grade 8 immersion classroom where the teacher effectively negotiated form with students by providing feedback, asking questions, and initiating discussions about language use, and where students negotiated form amongst themselves in cooperative learning activities with an analytic focus.

Résumé. Cet article soutient que la pédagogie immersive pourrait tirer profit de la négociation de la forme. En tant que stratégie analytique, la négociation de la forme diffère de la négociation du sens en ce qu'elle encourage les élèves à ne pas se contenter de simplement transmettre leur message. En poussant les élèves à produire des énoncés plus précis et plus appropriés, on cherche à les faire puiser dans leurs propres ressources (socio)linguistiques. Cet article présente quelques exemples d'une telle stratégie, tous tirés d'une classe d'immersion (8e année), où le professeur a efficacement négocié la forme avec ses élèves par le biais de rétroaction, de questions, et de discussions sur l'emploi de la langue. De plus, les élèves négociaient la forme entre eux par le biais d'activités analytiques en groupes d'apprentissage coopératif.
\end{abstract}

\section{Introduction}

Cet article a pour but de mettre en valeur le rôle que peut jouer la négociation de la forme dans la pédagogie en classe d'immersion. La négociation de la forme sera d'abord située dans un cadre théorique qui souligne l'importance du lien dans l'apprentissage d'une langue seconde entre la production langagière et la rétroaction et, de plus, qui favorise l'intégration des stratégies analytiques dans le contexte expérientiel de l'immersion. Ensuite, cette stratégie sera décrite grâce à des interactions entre professeur et élèves ainsi qu'entre élèves, telles que notées lors d'un projet de recherche entrepris en classe d'immersion au niveau de la huitième année. Dans un article précédent (Lyster 1990), il a été question de justifier l'intégration des stratégies d'enseignement analytique en classe d'immersion; le présent article vise à démontrer quelques exemples d'une stratégie analytique, à savoir la négociation de la forme, ainsi que son lien possible avec l'expérientiel.

\subsection{De la négociation du sens à la négociation de la forme}

La négociation du sens, terme employé pour caractériser l'interaction communicative entre parent et enfant dans une conversation, est considérée importante non seulement dans le développement de la langue maternelle mais aussi dans l'apprentissage en général (ex. : Wells 1981, 1985, 1986). Puisque les interactions aboutissent à une entente, c'est-à-dire à une compréhension mutuelle en dépit de lacunes d'ordre linguistique ou de limites cognitives, le

\footnotetext{
${ }^{1}$ Une première version de cet article a été présentée au Congrès de l'ACPI tenu à Régina en novembre 1992 et une deuxième version au Congrès de l'AQEFLS tenu à Montréal en mars 1993. Je tiens à remercier Jacques Rebuffot et Merrill Swain de leurs précieux conseils à propos de la présente version. J'aimerais également remercier 'Serge' et ses élèves de m'avoir accueilli dans leur salle de classe.
} 
concept de négociation du sens s'emploie également pour caractériser l'interaction en classe de langue seconde (ex. : Long et Porter 1985) et à la fois l'enseignement immersif (Snow 1989).

Pour caractériser la négociation du sens en classe d'immersion, Snow (1989:41-43) énumère d'abord quelques stratégies de communication employées par les professeurs d'immersion dans le but de faire comprendre leur message. Selon Snow, ils modifient leur production orale afin de la rendre plus compréhensible; il se peut qu'ils parlent plus lentement et plus clairement et, avec les élèves les plus jeunes, qu'ils utilisent des phrases plus courtes et moins complexes ainsi qu'un vocabulaire régulièrement réutilisé. Ils ont diverses manières de contextualiser leurs messages : la mimo-gestuelle, le mime, les supports visuels (cartes, photos, dessins, etc.), et le vécu même des élèves. De plus, ils ajoutent des éléments redondants à leur emploi de la langue par le biais de synonymes, de périphrases, de répétitions, et d'exemples. Ils rendent, également, prévisible le déroulement de la leçon en l'organisant en diverses étapes (routines d'ouverture et de fermeture, travail individuel et en groupe, devoirs, révisions).

Ensuite, quant à la compréhension du message des élèves, Snow constate que les professeurs doivent interpréter les énoncés des élèves en recourant à des vérifications : ils demandent aux élèves de répéter des informations, de développer certains points, de poser des questions à d'autres élèves, ou de résumer les idées principales de leurs énoncés. De plus, ils doivent leur enseigner des stratégies de communication pour qu'ils puissent se débrouiller lorsqu'ils ne comprennent plus, en posant des questions du type: "Pourriez-vous répéter?/Pourriez-vous me donner un autre exemple », etc. Enfin, Snow souligne l'importance de créer en classe d'immersion un environnement non seulement axé sur la compréhension mais également sur la production afin de permettre aux élèves d'utiliser souvent la langue seconde.

L'importance de la production langagière en classe d'immersion est également soulignée par d'autres chercheurs tels que Harley, Allen, Cummins et Swain (1990). On pourrait toutefois remettre en question le rôle de la négociation du sens lorsque celle-ci sert exclusivement à se faire comprendre. Par exemple, dans le cas des élèves en immersion, Harley (1984) signale que leur compétence stratégique, à savoir leur capacité à réussir la communication malgré des lacunes linguistiques, se développe bien mais peut éventuellement nuire à l'acquisition de la compétence grammaticale. De même, s'agissant de la négociation du sens en classe d'immersion, Swain (1985) dit qu'il ne suffit pas de se contenter des interactions qui débouchent sur une compréhension mutuelle en dépit d'une production fautive de la part des élèves. Selon elle, la compréhension exige une analyse sémantique de la part des élèves alors que la production les pousse à analyser la langue syntaxiquement. C'est, en effet, la production langagière qui leur permet de mettre à l'épreuve leurs hypothèses concernant la syntaxe et c'est la rétroaction du professeur et des pairs qui les pousse non seulement à se faire comprendre mais aussi à s'exprimer de manière précise, cohérente et appropriée. Afin que les élèves puissent bénéficier de ce processus à la fois interactif et heuristique, il faudrait donc que le professeur négocie non seulement le sens avec ses élèves, mais aussi la forme. À la lumière de cette proposition, nous voyons, dans ce que nous appelons la négociation de la forme, une stratégie analytique propice à la pédagogie immersive.

\subsection{La dimension analytique-expérientielle}

Selon Stern (1990, 1992), les stratégies analytiques sont celles qui visent l'usage correct de la langue des points de vue phonologiques, grammaticaux, fonctionnels, discursifs et sociolinguistiques. Elles mettent en évidence les traits langagiers qui sont étudiés selon des 
règles et des relations systématiques. Ces traits sont mis en pratique en vue d'une utilisation éventuelle. De leur côté, les stratégies expérientielles traitent de divers sujets et thèmes intéressants, visent à engager les élèves dans des activités signifiantes, et mettent l'accent sur la communication du message et l'emploi authentique de la langue. En outre, Stern souligne l'importance de considérer la dimension analytique-expérientielle comme un ensemble continu et non pas comme une dichotomie. Il prône l'intégration des stratégies analytiques et expérientielles dans des proportions qui varient en fonction des besoins des apprenants et des objectifs du programme. Il suggère, par exemple, que les programmes de français de base qui se préoccupent essentiellement de l'analyse de la langue devraient s'orienter davantage vers les stratégies expérientielles, alors que les programmes d'immersion devraient renforcer davantage l'enseignement expérientiel par des stratégies analytiques. ${ }^{2}$

L'enseignement analytique de la langue n'est pas nouveau dans les classes d'immersion, mais il se limite, selon certaines recherches (ex. : Swain et Carroll 1987), à l'enseignement de la grammaire décontextualisée. Il semble que l'on ait tendance en immersion à percevoir l'enseignement analytique et l'enseignement expérientiel comme opposés plutôt que complémentaires : un jour on enseigne les temps du passé et, le lendemain, on enseigne un cours d'histoire, sans faire de lien explicite entre les deux leçons, comme si l'étape de l'application (ou de la mise en pratique) qui devrait suivre une leçon de grammaire se déroulait automatiquement et naturellement en classe d'immersion.

Quel serait donc le lien entre une approche analytique et une approche expérientielle? Allen (1983) soutient que la médiation entre une approche structuro-analytique et une approche non-analytique pourrait être fournie par une approche fonctionnelle-analytique. Selon Stern (1992), 'l'analyse fonctionelle' met en effet en évidence :

1) le contexte

2) le rôle des participants (l'identification des interlocuteurs et le rapport entre eux)

3) le thème ou le sujet

4) la variation sociostylistique

5) la sémantique (y compris les intentions et la force illocutoire)

6) l'identification des actes de parole

7) l'analyse du discours

Il s'agit donc d'une approche communicative qui tend vers l'analyse du discours et des aspects sociolinguistiques ainsi que vers la mise en pratique des fonctions langagières.

L'effet d'un enseignement fonctionnel-analytique sur la compétence sociolinguistique des élèves en classe d'immersion a été investigué dans le cadre d'un projet de recherche rapporté dans Lyster (1993,1994). Il s'agissait de l'implantation dans trois classes d'immersion au niveau de la huitième année d'un matériel fonctionnel-analytique (Lyster 1991) où il était surtout question de la variation sociostylistique, à savoir l'étude et la mise en pratique de différents niveaux de langue. C'est dans cette perspective que le présent article présente quelques interactions et activités langagières observées dans une des classes où la négociation de la forme s'est avérée particulièrement efficace : d'abord comme stratégie utilisée par le professeur (que

\footnotetext{
${ }^{2}$ Voir également Rebuffot (1993) à propos de la dimension analytique-expérientielle en classe d'immersion.
} 
nous appellerons 'Serge') lorsqu'il communiquait avec ses élèves et, ensuite, comme stratégie utilisée par les élèves lors d'activités d'apprentissage coopératif.

\section{La négociation de la forme dans la classe de Serge}

Serge a été choisi parmi les trois professeurs parce que ses élèves et lui semblaient à l'aise dans l'approche fonctionnelle-analytique. De plus, ses élèves avaient évalué de façon positive le matériel pédagogique utilisé, mais aussi l'avaient considéré plus difficile que les autres classes. Ils étaient donc plus que les autres poussés pour répondre aux attentes de Serge. En fait, les observations faites dans sa classe ont montré que Serge était exigeant et qu'il en découlait des interactions linguistiquement riches. Quelques-unes de ces interactions seront présentées afin d'illustrer la négociation de la forme sous quatre aspects : 1) la rétroaction fournie par Serge à ses élèves; 2) les questions que Serge posait à ses élèves; 3) les discussions sur l'emploi de la langue; et 4) les activités d'apprentissage coopératif des élèves. Serge a consacré 18 heures échelonnées sur six semaines à l'enseignement de l'unité. Il a été observé pendant 14 heures.

\subsection{La rétroaction}

Serge fournissait au moins deux sortes de rétroaction différentes à ses élèves afin de négocier la forme avec eux : il exigeait plus de précision de leur part et leur demandait aussi d'adapter leurs productions aux contextes d'emploi. Analysons d'abord son exigence de précision. Dans l'exemple suivant, il vient de demander aux élèves d'indiquer les différences entre une correspondance officielle et une correspondance à caractère privé :

Élève 1: La chose en bas. ${ }^{3}$

Serge: « La chose en bas?! ».

Élève 1: La salutation finale.

Serge aurait pu facilement accepter la première réponse de l'élève ("La chose en bas"), en disant, par exemple, «Oui, c'est bien. C'est la salutation finale». Il lui aurait ainsi, par la même occasion, fourni le mot juste. Mais, en répétant, sur un ton surpris et réprobateur, la périphrase nébuleuse de son élève, Serge refuse d'accepter un énoncé vague. Il l'amène ainsi à utiliser le terme exact. La correction et la précision de la production lexicale par l'élève démontrent que ce dernier connaissait le terme en question mais attendait d'être poussé par l'enseignant pour le produire.

Passons à un autre exemple. Serge vient de faire écouter aux élèves une bande sonore décrivant la préparation d'une mousse au chocolat:

Serge: De quoi est-ce qu'on parle?

Élève 1: Une mousse au chocolat.

Serge: Mais pourquoi?

Élève 2: On donne des directions pour faire une mousse au chocolat.

Serge: Comment est-ce qu'on appelle ça?

Élève 3: Une recette.

\footnotetext{
${ }^{3}$ Les élèves sont numérotés (Élève 1, Élève 2, et ainsi de suite) afin d'identifier les différents locuteurs à l'intérieur d'une même interaction. Ainsi, l'élève qui parle plus d'une fois dans la même interaction est toujours identifié par le même chiffre. Cependant, il n'y a pas de lien identificateur d'une interaction à l'autre.
} 
Lorsque l'élève répond qu'il s'agit de donner « des directions» pour faire une mousse, l'enseignant aurait pu encore une fois répondre : "Oui, c'est une recette ». Mais le fait que Serge refuse d'accepter de telles périphrases oblige ses élèves à produire le mot juste. C'est une technique qui fait appel aux ressources cognitives de l'apprenant. Par contraste, on peut comparer la correction des erreurs observée chez un professeur dans une autre classe où on commente un extrait tiré du roman Le cave (traduction française du roman américain Wilted (Kropp 1981)). Il s'agit d'une jeune fille qui présente son ami à sa mère pour la première fois; la discussion traite du tutoiement et du vouvoiement:

Prof: $\quad[\ldots]$ Autre chose?

Élève 1: Ils savent ...

Prof: Ils se connaissent.

Élève 2: Ils sont le même âge.

En fournissant la forme correcte, sans indiquer qu'il y a une erreur et sans encourager l'élève à reformuler son énoncé, ce professeur ne fait pas prendre conscience à l'Élève 1 de son erreur. On pourrait même supposer que, lorsqu'il réplique "Ils se connaissent ", cet élève est amené à croire que l'enseignant renforce son énoncé en lui fournissant un synonyme, comme cela se fait souvent dans un cours de langue. ${ }^{4}$ Il n'y a donc pas ici d'appel à la réflexion de l'élève qui reste ainsi passif.

Comment cet enseignant aurait-il pu négocier plus efficacement les formes du message et leur sens? Par exemple, lorsque l'élève répond «Ils savent», il aurait suffi de demander : « Ils savent quoi? Qu'est-ce que tu veux dire par là? Qu'est-ce qu'ils savent? », et ainsi de suite.

La deuxième façon que Serge utilisait pour fournir la rétroaction à ses élèves était de les pousser à produire des énoncés plus appropriés au contexte. Par exemple, dans un premier temps, il fait l'exercice suivant (adapté de Astley et Hawkins (1985) avec la permission de l'éditeur), en demandant aux élèves de suggérer des répliques plus appropriées aux contextes:

Dans les deux contextes suivants, essaie de suggérer une réplique plus vraisemblable:

a) Contexte 1 : Un garçon au lit; sa mère est inquiète.

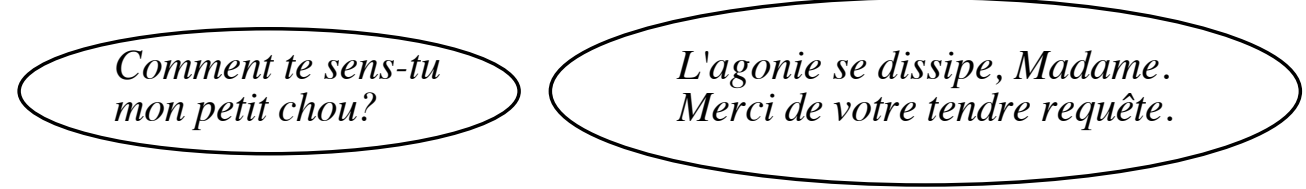

b) Contexte 2: Une patronne à son bureau; un employé lui fait face.

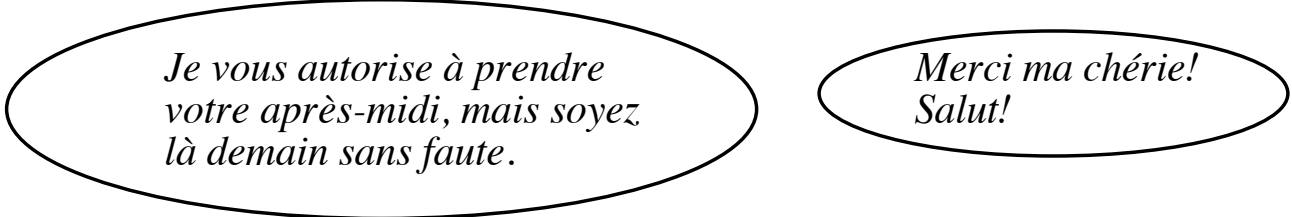

${ }^{4}$ De plus, croire que les verbes français 'savoir' et 'connaître' sont synonymes est une idée fausse que se font de nombreux anglophones apprenant le français. 
Suite au deuxième contexte, Serge demande aux élèves de suggérer une réplique plus convenable:

Élève 1: Merci Madame.

Élève 2: Je te remercie.

Serge: « Je te remercie? ». Est-ce qu'on peut faire un changement?

Élève 2: Je vous remercie.

Serge: Pourquoi?

Élève 2: C'est plus poli.

Serge ne corrige pas la suggestion de l'Élève 2 («Je te remercie ») car elle n'est pas forcément fausse. Tout de même, il lui demande d'abord s'il est possible de modifier l'énoncé et d'expliciter ensuite l'effet d'une telle modification.

Dans les exemples suivants tirés de certains jeux de rôles, Serge continue à encourager des énoncés plus appropriés aux divers contextes exigés par le rôle des participants:

Exemple 1 (jeu de rôles entre deux dames qui ne se connaissent pas) :

Élève 1: Peux-tu m'indiquer où se trouve le poste de police?

Serge: Vous n'êtes pas très polie, Madame.

Élève 1: Pourriez-vous m'indiquer où se trouve le poste de police?

Élève 2: Je ne sais pas.

Serge: Ce n'est pas une réponse très polie.

Élève 2: Je regrette, Madame, je ne sais pas où se trouve le poste de police.

Exemple 2 (un élève qui s'adresse à une auteure connue, Suzanne Martel) :

Élève 1: Est-ce que cela vous conviendrait de venir parler à la classe de français mardi?

Élève 2: Non, je suis occupée.

Serge: Aimes-tu sa réponse?

Élève 1: Non.

Serge: Donne une autre réponse plus polie.

Élève 2: C'est dommage, mais je suis occupée.

Exemple 3 (entre deux amis) :

Élève 1: Veux-tu faire de la plongée sous-marine?

Élève 2: Oui, merci.

Serge: Sois réaliste!

Ainsi, malgré la perspective analytique qui sous-tend ces jeux de rôles, Serge tente de maintenir un certain degré de vraisemblance dans ces contextes artificiels.

\subsection{Le questionnement}

De plus, Serge posait souvent des questions à partir des réponses précédentes des élèves, ce qui les poussait à approfondir ou à préciser leurs énoncés. Ainsi, ce genre de négociation se rencontre d'abord dans une discussion qui a lieu au moment de la sensibilisation de la classe aux niveaux de langue. L'interaction se fait au sujet de la bande dessinée suivante, tirée de Astley et Hawkins (1985) (avec la permission de l'éditeur) : 


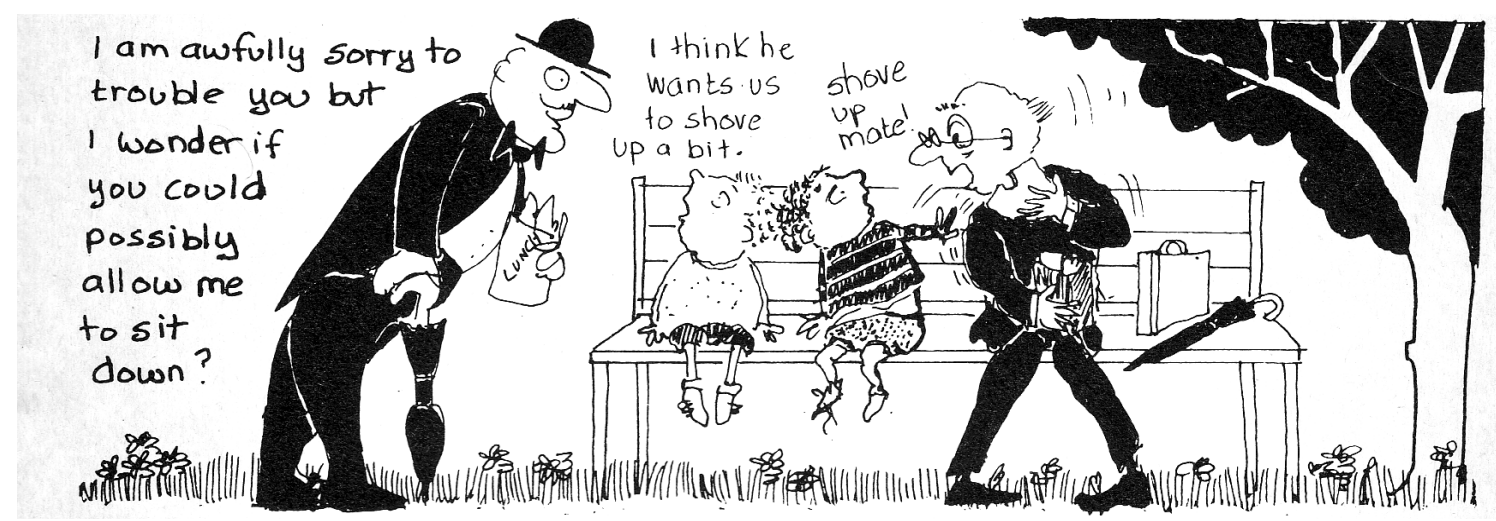

L'emploi de l'anglais dans la bande dessinée sert de point de départ pour l'analyse des niveaux de langue en français. Le but est de partir du vécu des élèves et de leurs connaissances sociolinguistiques qui sont déjà bien développées, ainsi que nous le démontre l'interaction suivante:

Serge: Qu'est-ce qu'il a de bizarre, le monsieur?

Élève 2: Il est trop poli.

Élève 3: Le monsieur le dirait à un adulte.

Serge: N'importe quel adulte?

Élève 4: Moi je le dirais à quelqu'un qui est riche.

Élève 5: À quelqu'un que tu ne connais pas.

Élève 6: Quelqu'un que tu respectes.

Serge: Est-ce que ta mère le dirait à ton père?

Élève 6: Peut-être, si elle voulait être sarcastique.

Le questionnement employé par Serge durant cette interaction est digne d'attention. Lorsque l'Élève 3 constate que l'homme aurait dû s'adresser de façon soutenue plutôt à un autre adulte qu'à un enfant, Serge demande des précisions supplémentaires. En effet, même s'il est vrai que l'homme aurait pu s'adresser ainsi à un autre adulte, et non pas à un enfant, il n'aurait probablement pas parlé de cette façon à n'importe qui : cela dépend du degré de familiarité entre les interlocuteurs. Voilà où Serge veut en venir lorsqu'il demande " N'importe quel adulte? ». Cette dernière question obtient une réponse plutôt drôle ("quelqu'un qui est riche »), et une autre qui est plus juste (« quelqu'un que tu ne connais pas »). Lorsque l'Élève 6 indique qu'il est question de respect, Serge insiste pour avoir des précisions afin d'éviter le faux raisonnement suivant : on s'adresse de manière soutenue à quelqu'un parce qu'on respecte la personne, donc on ne respecte pas la personne à qui on s'adresse de manière familière. Ainsi, dans le but de faire comprendre qu'un discours familier ne manque pas forcément de respect, Serge demande à l'élève si une femme mariée aurait parlé de manière si soutenue à son époux (prenant pour acquis qu'il y a du respect entre les époux!). Ce genre de questionnement permet aux élèves de partager leurs connaissances déjà acquises et de faire preuve, en fait, d'une conscience sociolinguistique assez avancée.

De même, l' interaction suivante illustre bien les questions employées par Serge dans le but de pousser les élèves à décrire certains traits sociostylistiques en français et, en fin de compte, à pouvoir se corriger entre eux. Il s'agit d'une comparaison de deux textes écrits décrivant tous les deux la préparation d'une mousse au chocolat : le premier est une note 
adressée à quelqu'un de connu; le second une recette dans un livre de cuisine. On lit d'abord la note et ensuite la recette:

Serge: C'est quel niveau?

Élève 1: Formel.

Serge: Quel est le contexte?

Élève 2: C'est dans un livre de recettes.

Serge: Pourquoi formel?

Élève 3: On dit « les sucrer».

Serge: À la place de quoi?

Élève 3: « Mets du sucre ».

Élève 4: Ils utilisent l'infinitif.

Serge: Donne-moi des exemples.

Élève 4: « mettre », « monter », « faire », « incorporer »...

Serge: Qu'est-ce qu'il y a dans le texte informel?

Élève 5: « Mets », « fais », « ajoute »...

Serge: C'est quel temps ça?

Élève 5: Le futur.

Élève 6: Non, c'est le présent—c'est l'impératif.

Il convient de noter le débit de cette interaction comportant de nombreuses questions suivies de réponses justes qui démontrent les connaissances que certains élèves partagent avec les autres.

De plus, à maintes reprises Serge pose la question « Pourquoi? » pour inciter les élèves à justifier leurs réponses. Lorsque certains élèves ne sont pas d'accord, Serge leur demande des explications pour que leurs réponses renseignent les autres. Par exemple, lorsqu'il est proposé que «Salut! À demain! » est une forme soutenue à cause de ‘à demain', une élève fait remarquer qu'il s'agit d'une forme plutôt familière car, dit-elle, «On dit pas 'Salut' à une personne importante ».

\subsection{Les discussions à propos de l'emploi de la langue}

Au-delà de la rétroaction et du questionnement, Serge engageait des discussions à propos de l'emploi de la langue pour faire réfléchir les élèves à la communication. La négociation de la forme (et du niveau de langue) était encore évidente dans certaines discussions qui suivaient des présentations orales. Nous en présentons maintenant un exemple : les élèves ont à présenter d'abord des sketches à partir de deux contextes différents, l'un dans un magasin où client et vendeur sont en désaccord et l'autre, dans le cadre d'une discussion télévisée. Les sketches sont enregistrés au magnétoscope pour permettre aux élèves de se corriger. Mais, par la suite, Serge, allant plus loin que la correction des erreurs, demande aux élèves comment les aspects sociolinguistiques changeraient dans d'autres contextes et avec d'autres interlocuteurs. Ainsi, à la suite d'un sketch où il s'agit d'une entrevue entre un animateur et un chanteur de rock qui se vouvoient, Serge demande à la classe ce qui changerait si l'animateur était Arsenio Hall. Les élèves tombent alors d'accord pour dire qu'en français il aurait tutoyé le chanteur à cause de son style très familier.

Dans la classe de Serge, d'autres discussions à propos de l'emploi de la langue, toujours dans une perspective analytique, se déroulaient aussi sur les thèmes suivants : 1) la manière dont les élèves s'adressent à des professeurs à l'intérieur et à l'extérieur de la classe (ex. : le tutoiement ou le vouvoiement); 2) les différentes variétés du français (à savoir les différences 
entre le français du Québec et celui de France), ainsi que les différentes variétés de l'anglais dans le monde par rapport à l'anglais parlé au Canada; 3) l'emploi des jurons et le mélange des niveaux de langue. Dans la même perspective, enfin, Serge leur demandait de compléter plusieurs exercices où il fallait classer des énoncés (soutenus, familiers ou neutres) et ensuite justifier les réponses.

\subsection{Les activités d'apprentissage coopératif}

Alors que la rétroaction, le questionnement et les discussions décrits ci-dessus font partie de la négociation de la forme entre le professeur et ses élèves, les activités décrites ci-dessous nécessitent la négociation entre élèves, toujours dans une perspective analytique. Tel que l'on peut le constater dans le tableau 1, la disposition des pupitres dans la classe de Serge est propice au travail d'équipe : les 28 élèves sont repartis en sept groupes de quatre.

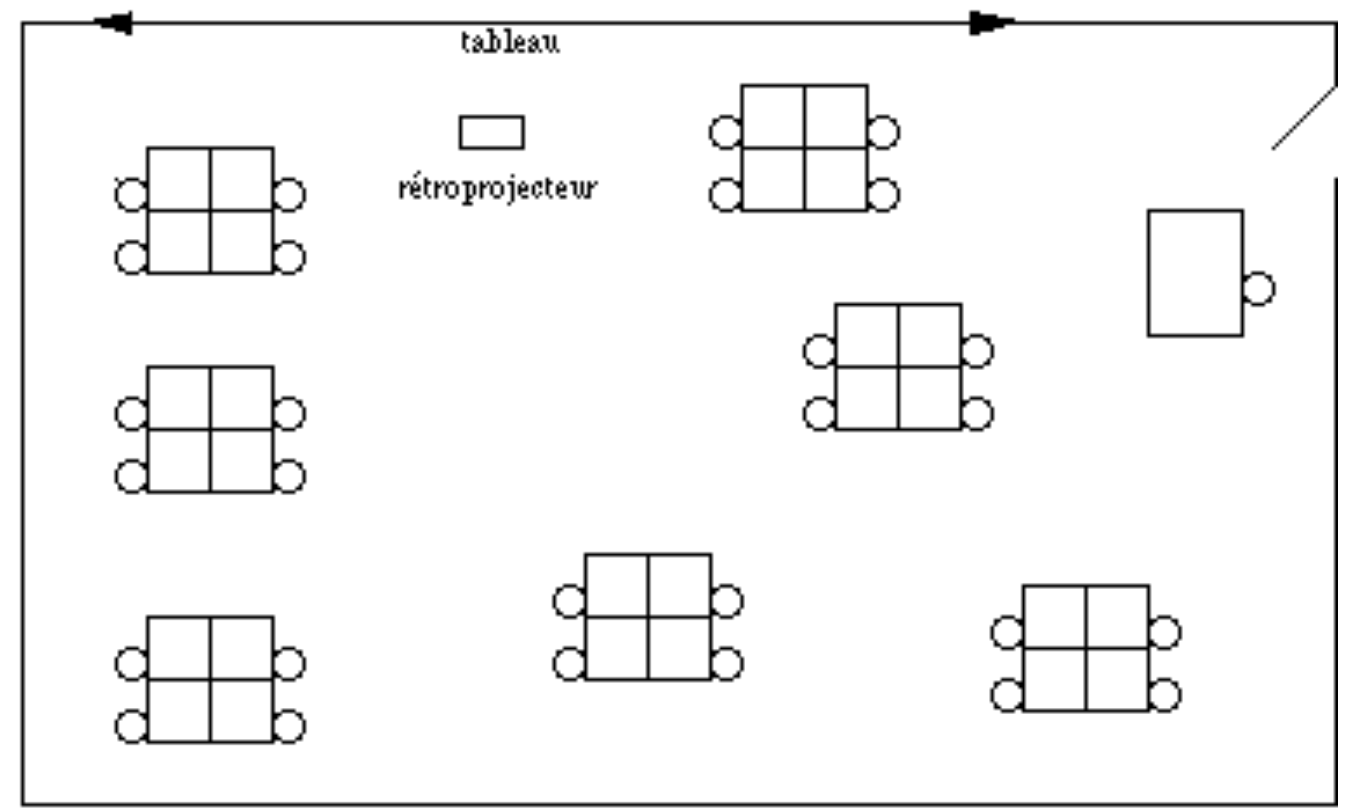

Tableau 1 : La disposition des élèves dans la classe de Serge

La première activité consiste à corriger en équipe des lettres écrites par les élèves à l'occasion d'un échange fictif entre une école en Ontario et une école au Québec (voir Annexe A). Dans un premier temps, chacun doit écrire une lettre, soit à sa famille d'accueil, soit à la direction de l'école à fréquenter durant l'échange. Dans un deuxième temps, insatisfait de la qualité des lettres que ses élèves lui remettent, Serge les leur rend en exigeant qu'ils corrigent les lettres des uns et des autres en groupes de quatre dans le but d'en améliorer la qualité. Le premier élève doit corriger le genre, le deuxième l'emploi des verbes, le troisième l'orthographe, et le quatrième le message et les niveaux de langue. Selon Serge, ce processus de correction collective améliore nettement la qualité des lettres et pousse les élèves à utiliser le français, ce qui a été confirmé par nos observations.

En outre, Serge organisait une série d'activités d'apprentissage coopératif, selon la méthode Jigsaw, pendant cinq heures échelonnées sur un peu plus d'une semaine dans la classe de français. Ces activités avaient pour but de mettre en évidence les différences sociostylistiques entre l'emploi soutenu et l'emploi familier du français, et entre l'écrit et l'oral. La méthode 
Jigsaw nécessite la création de groupes d'origine, dont chacun des quatre membres devient ensuite membre d'un différent groupe dit d'experts. Les membres de chaque groupe d'experts deviennent « experts » dans un certain domaine et puis retournent dans leurs groupes d'origine afin de partager ce qu'ils ont appris dans leur domaine d'expertise (voir Annexe B).

Serge entame l'activité avec la classe entière par une présentation de quatre textes exprimant le même message, à savoir comment enregistrer avec un magnétophone, mais dans quatre styles différents convenant à quatre contextes différents : deux à l'oral, deux à l'écrit. Après avoir fait écouter les textes enregistrés sur cassette et fait lire les textes écrits, Serge interroge les élèves sur l'identité des participants et le but du message. À l'oral il s'agit d'un contexte d'affaire où un vendeur s'adresse à une cliente dans un magasin et, ensuite, d'un contexte familier entre deux amis. À l'écrit, il s'agit d'abord d'un texte tiré du manuel d'utilisation du magnétophone, puis d'une note écrite à un ami.

$\mathrm{Au}$ sein des groupes d'origine, les élèves comparent, ensuite, le vocabulaire et les expressions des quatre textes. Par exemple, ils doivent trouver quatre mots dans le texte soutenu correspondant au mot 'piton' utilisé dans la note familière (le commutateur, le sélecteur, la touche, le bouton), et quatre mots ou expressions utilisés dans le texte soutenu correspondant au verbe passse-partout 'mettre' dans la note familière (placer, insérer, faire glisser, remettre). Puis, suite à cet exercice, chaque membre du groupe d'origine doit choisir son domaine d'expertise : l'écrit soutenu, l'écrit familier, l'oral soutenu, ou l'oral familier.

Serge présente, ensuite, à la classe une deuxième série de quatre textes différents décrivant la préparation d'une mousse au chocolat. En tant que membres de groupes d'experts, les élèves entreprennent alors une comparaison détaillée des textes en fonction de leur domaine d'expertise. Par exemple, les élèves chargés d'écrire un texte soutenu se concentrent davantage sur ce qui caractérise ce genre de texte par rapport aux autres textes. Une fois terminé cet exercice de comparaison, les « experts » retournent en groupes d'origine où ils procèdent à la correction par le biais d'une négociation leur permettant de découvrir une structure d'interdépendance reliant les réponses trouvées par chaque expert.

Suite à une brève présentation d'une troisième série de quatre textes ayant pour but de convaincre quelqu'un d'acheter un ordinateur, Serge demande alors à chaque groupe d'origine de choisir un message devant être exprimé dans quatre contextes différents suivant le modèle déjà présenté : deux textes écrits (l'un soutenu et l'autre familier) et deux textes oraux (soutenu et familier) enregistrés sur cassette. L'expert en oral familier, par exemple, doit s'occuper surtout du texte familier à l'oral, mais les quatre membres du groupe doivent d'abord s'entendre sur le texte de base, en négociant le thème et les quatre contextes ainsi que les traits sociostylistiques à mettre en valeur. Lors de la présentation des textes devant la classe (la lecture des textes écrits et l'écoute des textes oraux sur cassette), les élèves doivent utiliser le niveau de langue qui convient au contexte et être très précis dans leur production. De plus, chaque membre du groupe doit décrire le contexte du message et énumérer au moins quatre caractéristiques qui rendent le message représentatif d'un certain niveau et du code oral ou du code écrit, selon le cas. Les thèmes choisis par les différents groupes sont les suivants : indiquer le chemin à Québec dans une invitation orale ou écrite; présenter les règlements de la salle de classe; demander quelques articles dans un grand magasin; préparer un banana split, du maïs soufflé au four à micro-ondes, et des biscuits aux brisures de chocolat. 
On peut tirer d'importantes conclusions de l'analyse du travail en équipe effectué par Serge. Alors que l'analyse du vocabulaire et des niveaux de langue était évidemment très analytique, les élèves de cet enseignant négociaient la tâche en français, aboutissant donc à une activité à la fois analytique et expérientielle. Nous en concluons que les activités analytiques pourraient se dérouler plus souvent en groupes d'apprentissage coopératif afin d'aboutir à une pédagogie intégrative. Même si on a souvent considéré qu'une activité d'apprentissage coopératif convenait bien au cours d'histoire ou à l'étude d'un roman et moins bien à l'analyse de la langue, selon nos observations en salle de classe et étant donné les diverses connaissances linguistiques des élèves en immersion, il nous paraît tout à fait possible que les élèves en immersion puissent procéder à l'analyse de la langue par le biais d'activités d'apprentissage coopératif. Il est intéressant de noter les commentaires que les élèves ont écrits pour répondre, entre autres, à la question suivante : «En général, crois-tu que le travail en groupe peut t'aider à améliorer ton français? Pourquoi? ». Les élèves avaient le choix de répondre en français ou en anglais au questionnaire anonyme :

- Oui, on peut "discuss" et "share" nos idées et les comparées entre nous même.

- Oui, parce que tu peux avoir, écouter, et comprendre les suggestions des autres.

- Oui parce que quand tu fait un faut ton groupe peut t'aider.

- Oui parce que quand tu travaille dans un groupe les idée entour toi est sa te donne une meilleur note.

- Oui parce que sa t'enseigne de cooperate.

- Oui parce que le groupe peuvent te aider et parler en français et te corriger ton faut de grammar quand tu fais le travaille.

- Oui, je pense que le travail en groupe était bon parce que tu peux avoir les differentes opinion de français. Mais quand tu travail en groupe je trouve que vous devez être três patient.

- Oui, parce que tous le mond doit apprendre comment travailer en group pour le future.

- Yes, because in groupes you can help each other more. When you have to work alone, you can only go to the teacher for help, and some people don't like to do that.

- Oui parce que ça m'aide à parler plus clairement dans un groupe en français.

- Ca peut t'aider un peu, parce que ça vous donne de pratique en français et les autres dans la groupe peut vous aider à corriger vos fautes.

- Oui parce qu'il y a plus de personne à pensait a des chose.

- Oui parce que quand je ne sais pas un réponse je peux demander ma groupe de l'aide.

- Oui, parce que si tu fait des erreurs, les autres personnes peux t'aider, ou te corriger.

L'unanimité de ces évaluations positives, ainsi que les observations qui ont confirmé que les élèves négociaient entre eux en français, mettent en évidence le rôle que peut jouer l'apprentissage coopératif en tant que médiateur entre les stratégies analytiques et expérientielles. 


\subsection{Et enfin, un peu d'humour ...}

Avant de conclure, il convient de mentionner quatre autres traits qui semblent avoir contribué au bon rapport que Serge avait avec ses élèves, bien qu'il n'ait eu que deux ans d'expérience à l'époque des observations :

1) Serge participait aux jeux de rôles avec les élèves.

2) il racontait des anecdotes personnelles très pertinentes. De même, il encourageait les élèves à raconter leurs propres expériences reliées aux activités (par exemple, leurs visites au Québec ainsi que les échecs et les succès de la communication).

3) Serge intégrait bien quelques mini-leçons sur la grammaire au bon moment selon les besoins communicatifs des élèves.

4) Serge avait un sens de l'humour développé : il comprenait suffisamment bien les aspects sociolinguistiques en question pour pouvoir les exprimer de manière humoristique. Par exemple, au début de la première leçon il demandait à une élève, " Auriez-vous l'obligeance d'effacer le tableau, s'il vous plaît? » et puis à la suite du rire de cette élève il ajoutait, «Eh, efface-moi le tableau ». Il recherchait l'aide de quelques élèves en leur demandant, " Auriezvous l'obligeance de m'aider, s'il vous plaît? » et il concluait la leçon du jour par ces mots : « Mesdames et messieurs, merci beaucoup de m'avoir accordé une attention complète ».

À leur tour, les élèves semblaient prendre du plaisir à participer à des jeux de rôles. Par exemple, une jeune fille, qui jouait le rôle de la Présidente de l'Association des Parents, demandait un jour à un garçon de lui indiquer le chemin pour aller au gymnase. D'emblée, les élèves ont éclaté de rire parce que le gymnase se trouvait loin de la classe et que le chemin semblait assez compliqué. Lors de sa réponse, le garçon confondait sans cesse le tu et le vous, mais il était incité à se corriger par les commentaires et les rires de ses pairs. Il termina sa longue explication par : « ... et tu es là!... et vous es là! ...et vous êtes là! ». L'emploi correct de « vous êtes » a provoqué des applaudissements de la part des élèves. À ce moment-là, on a demandé par l'interphone à un certain élève, Steve, de se présenter au bureau du directeur Alors que Steve quittait la classe, celui qui venait d'indiquer le chemin à la " Présidente » s'est exclamé : « Steve, tu tournes droite... », ce qui a encore provoqué des rires. Par la suite, une autre élève a dû indiquer le chemin dans un contexte soutenu. Elle a réussi à dire vous de manière constante, bien qu'hésitante. Son succès a également provoqué des applaudissements.

\section{Conclusion}

En conclusion, on peut constater que Serge réussissait à intégrer les stratégies analytiques et expérientielles grâce à une certaine dynamique qui encourageait tantôt la communication, tantôt la réflexion sur cette même communication. Une telle dynamique était évidente dans les discussions sur l'emploi de la langue et dans les activités analytiques entreprises en groupes d'apprentissage coopératif qui encourageait la négociation entre les élèves. De même, la négociation générée par la rétroaction fournie par Serge et par son questionnement poussait ses élèves à réfléchir à ce qu'ils disaient et à s'exprimer de façon plus précise et plus appropriée.

Même si nous avons pu déceler un lien entre les stratégies analytiques et expérientielles, il importe toutefois de distinguer l'interaction de type expérientiel de l'interaction de type analytique dans l'enseignement en immersion. La première devrait continuer à jouer un rôle 
important en classe d'immersion de manière à y assurer diverses expériences langagières, alors que la dernière, dont la négociation de la forme fait partie, pourrait jouer un rôle secondaire de manière à affiner les compétences grammaticale et sociolinguistique des élèves.

La négociation de la forme, employée comme stratégie analytique, contribue à la résolution du problème qui sous-tend la correction des erreurs, à savoir comment corriger sans interrompre la communication. En effet, en ce qui concerne la négociation de la forme, il ne s'agit pas pour autant de la correction traditionnelle des erreurs qui consiste à interrompre l'élève qui reste passif, pour lui fournir le mot juste ou la bonne expression. Ce type de correction ne semble pas nécessaire (ni efficace) dans la mesure où nous avons pu constater que les élèves en immersion, au niveau de la huitième année, partagent entre eux suffisamment de connaissances linguistiques pour se corriger eux-mêmes et corriger les autres au moyen de la négociation, à condition d'être suffisamment encouragés à le faire. Sans encouragements, ils ont tendance à opter pour un discours simplifié, caractérisé par un vocabulaire restreint et un mélange de niveaux de langue, en s'attendant à ce que le professeur joue son rôle traditionnel de 'transmetteur de connaissances' et fournisse le mot juste. Or, en partant de ce que les élèves savent déjà et en les incitant à aller au-delà, la négociation de la forme s'avère une stratégie efficace dans l'enseignement et dans l'apprentissage du français en classe d'immersion dans la mesure où elle pousse les élèves à mieux s'exprimer. Ce faisant, elle ne décourage point la communication puisqu'elle en fait partie.

ROY LYSTER (Ph.D., Université de Toronto) est Professeur adjoint au Département de la didactique des langues secondes à l'Université McGill où il œuvre dans la formation des maîtres en français langue seconde. Il a enseigné plusieurs années en classe d'immersion avant de poursuivre en salle de classe ses recherches traitant de la pédagogie immersive.

\section{RÉFÉRENCES}

Allen, P. 1983. "A three-level curriculum model for second-language education," /La Revue canadienne des langues vivantes, 40(1):23-43.

Astley, H. and E. Hawkins. 1985. Using Language. Cambridge: Cambridge University Press.

Harley, B. 1984. “Mais apprennent-ils vraiment le français?" Langue et société 12 (Hiver):57-63.

Harley, B., P. Allen, J. Cummins and M. Swain. 1990. The Development of Second Language Proficiency. Cambridge: Cambridge University Press.

Heller, M., G. Barker et L. Lévy. 1989. "Projets de recherche et d'apprentissage coopératif : L'annonce publicitaire." Toronto: Centre de recherches en éducation franco-ontarienne, L'Institut d'études pédagogiques de l'Ontario.

Kropp, P. 1981. Le cave. Montréal: Fides.

Long, M. et P. Porter. 1985. "Group work, interlanguage talk, and second language acquisition," TESOL Quarterly 19(2):207-228.

Lyster, R. 1990. "The role of analytic language teaching in French immersion programs," $/ L a$ Revue canadienne des langues vivantes 47(1):159-176. 
Lyster, R. 1991. Variation en situation de communication: unité sur les niveaux de langue en français. Toronto: Conseil scolaire de Scarborough.

Lyster, R. 1993. "The Effect of Functional-Analytic Teaching on Aspects of Sociolinguistic Competence: A Study in French Immersion Classrooms at the Grade 8 Level." Thèse de doctorat. Université de Toronto.

Lyster, R. 1994 "The effect of functional-analytic teaching on aspects of French immersion students' sociolinguistic competence," Applied Linguistics. 15, 263-287

Rebuffot, J. 1993. Le point sur l'immersion au Canada. Montréal: Centre Éducatif et Culturel.

Snow, M. 1989. "Negotiation of meaning in the immersion classroom." In Negotiation of Meaning: Teacher's Activity Manual. Rockland, MD: Board of Education of Montgomery County.

Stern, H. H. 1990. "Analysis and experience as variables in second language pedagogy." In B. Harley, P. Allen, J. Cummins et M. Swain (réd.) pp. 93-109, The Development of Second Language Proficiency. Cambridge: Cambridge University Press.

Stern, H. H. 1992. Issues and Options in Language Teaching. Oxford: Oxford University Press.

Swain, M. 1985. "Communicative competence: some roles of comprehensible input and comprehensible output in its development." In S. Gass et C. Madden (réd.), Input in Second Language Acquisition. Rowley, MA.: Newbury House Publishers, Inc.

Swain, M. et S. Carroll. 1987. "The immersion observation study." In B. Harley, P. Allen, J. Cummins et M. Swain (réd.), Development of Bilingual Proficiency. Final Report. Volume II: Classroom Treatment. Toronto: Centre des langues modernes, Institut d'études pédagogiques de l'Ontario (O.I.S.E.).

Wells, G. 1981. "Language as interaction: The study of language development." In G. Wells (réd.) pp. 22-72, Learning through Interaction. Cambridge: Cambridge University Press.

Wells, G. 1985. "Language and learning: An interactional perspective." In G. Wells et J. Nicholls (réd.) pp. 21-39, Language and Learning: An Interactional Perspective. London: The Falmer Press.

Wells, G. 1986. The Meaning Makers: Children Learning Language and Using Language to Learn. Portsmouth, NH: Heinemann. 


\section{ANNEXE A : ÉCHANGE ONTARIO-QUÉBEC}

Félicitations! Tu as été accepté/e pour participer à un échange au Québec. Lis d'abord la lettre provenant du Secrétariat d'État, et ensuite celle de la famille d'accueil.

Secrétariat d'État

Gouvernement du Canada

Ottawa (Ontario)

le 15 février

M/Mlle

J'ai l'honneur de vous faire savoir que le Secrétariat d'État a approuvé votre demande de participer à l'Échange Ontario-Québec. Pendant la période allant du 1 mai au 31 mai vous assisterez aux cours de la Polyvalente Leduc, 23, avenue du Pont, Jonquière. Je vous prie de bien vouloir communiquer par écrit immédiatement avec la directrice de cette école, Mme LANGEVIN, pour lui indiquer votre présent niveau de scolarité, ainsi que les cours que vous suivez actuellement, afin d'assurer votre placement dans une classe appropriée.

Par ailleurs, vous serez hébergé pendant votre séjour chez la famille Bouchard, dont le domicile se trouve au 583, rue de la Bleuetière à Jonquière. À cet égard, je vous demande également de répondre à votre famille d'accueil afin de vous présenter et de confirmer votre arrivée, soit lundi le 30 avril à 23 h10 par Via Rail.

Vous trouverez votre billet de train ci-inclus. Comme vous pouvez le constater, le voyage aller-retour est pris en charge par le Gouvernement canadien.

Je vous prie d'agréer, M/Mlle , l'assurance de ma considération distinguée.

Pour le Secrétariat d'État:

Roland CAMBIER 
583 , rue de la Bleuetière

Jonquière (Québec)

le 20 février

Bonjour,

C'est avec beaucoup de plaisir que nous te recevrons chez nous cet été. Nos enfants ont bien hâte de faire ta connaissance, et nous prévoyons déjà un beau voyage à la campagne.

Écris-nous dès que possible afin de nous indiquer la date et l'heure de ton arrivée. Nous viendrons te chercher à la gare. Dans ta lettre, décris-toi d'abord pour que nous puissions mieux te connaître à la gare, et ensuite parle-nous de toi. Nous pourrons donc rendre ton séjour chez nous le plus agréable que possible.

Au plaisir de te recevoir,

M et Mme Bouchard

\section{ACTIVITÉS}

1. Écris à la directrice de l'école. Présente-toi en expliquant qui tu es et pourquoi tu lui écris. Dis-lui quel est ton niveau scolaire et décris-lui les cours que tu suis actuellement. Suis le modèle d'une lettre formelle, en te servant d'une salutation finale très formelle.

2. Écris à ta famille d'accueil pour te présenter et pour annoncer les détails de ton arrivée. Pour qu'ils te reconnaissent à la gare, tu dois te décrire: ton âge et ton apparence physique, y compris les vêtements que tu porteras. Décris également ta famille et l'endroit où tu habites, ainsi que ce que tu aimes faire en été. Suis le modèle d'une lettre formelle, mais sers-toi d'une salutation finale qui est moins formelle. Choisis parmi les suivantes :

"Au plaisir de vous rencontre ».

«Recevez mes sincères salutations ».

" J'ai hâte de faire votre connaissance ». 


\section{ANNEXE B : L'APPROCHE JIGSAW}

D’après Heller, Barker et Lévy (1989:18)

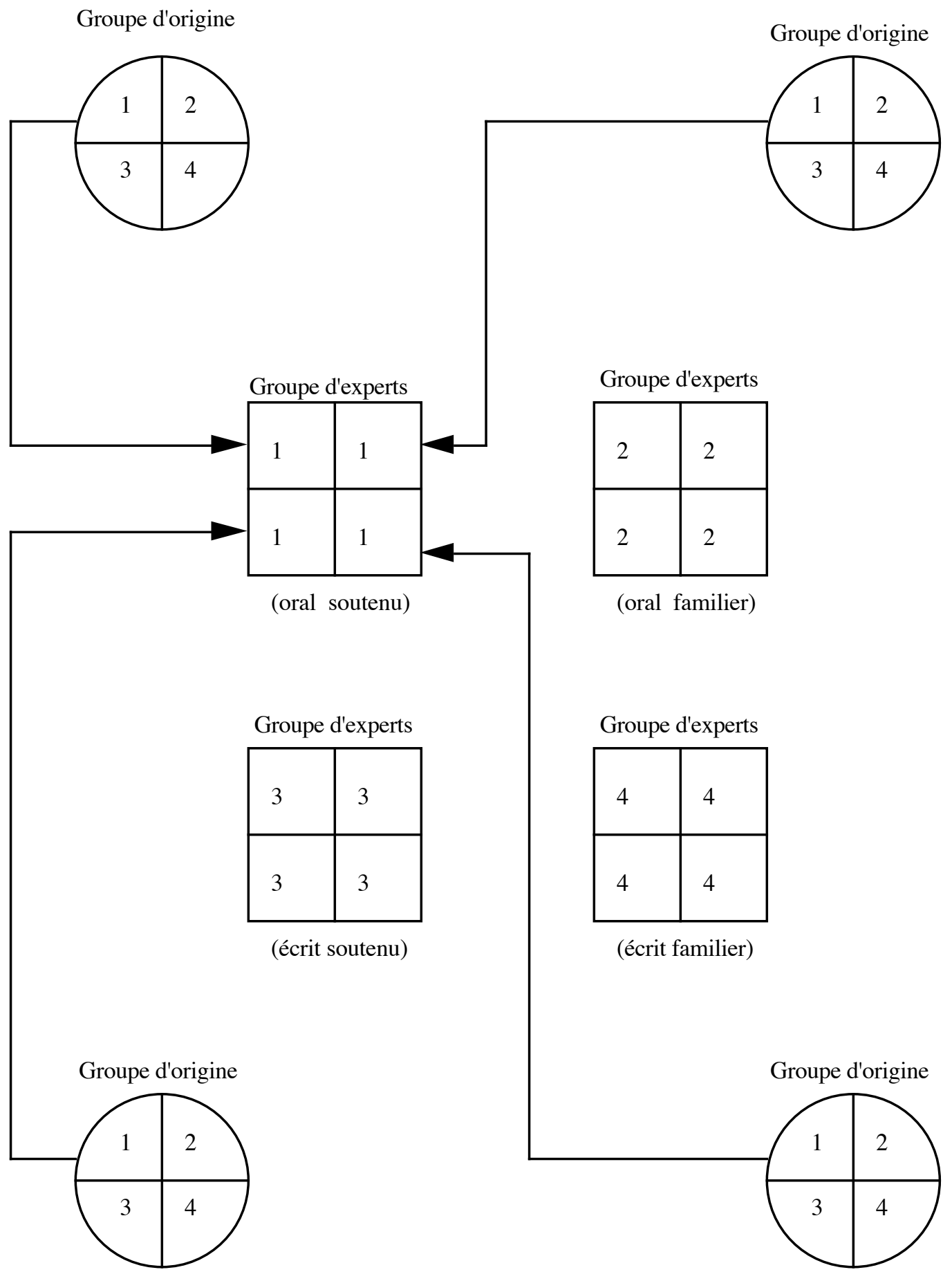

